\title{
Modifications Made to ModelMuse to Add Support for the Saturated-Unsaturated Transport Model (SUTRA)
}

Chapter 49 of

Section A, Groundwater

Book 6, Modeling Techniques

This Techniques and Methods report supplements USGS Techniques and Methods 6-A29

Techniques and Methods 6-A49 


\section{Modifications Made to ModelMuse to Add Support for the Saturated-Unsaturated Transport Model (SUTRA)}

By Richard B. Winston

Techniques and Methods 6-A49 


\title{
U.S. Department of the Interior SALLY JEWELL, Secretary
}

\section{U.S. Geological Survey Suzette M. Kimball, Acting Director}

\author{
U.S. Geological Survey, Reston, Virginia: 2014
}

For more information on the USGS - the Federal source for science about the Earth, its natural and living resources, natural hazards, and the environment, visit http://www.usgs.gov or call 1-888-ASK-USGS.

For an overview of USGS information products, including maps, imagery, and publications, visit http://www.usgs.gov/pubprod

To order this and other USGS information products, visit http://store.usgs.gov

Any use of trade, firm, or product names is for descriptive purposes only and does not imply endorsement by the U.S. Government.

Although this information product, for the most part, is in the public domain, it also may contain copyrighted materials as noted in the text. Permission to reproduce copyrighted items must be secured from the copyright owner.

Suggested citation:

Winston, R.B., 2014, Modifications made to ModelMuse to add support for the Saturated-Unsaturated Transport model (SUTRA): U.S. Geological Survey Techniques and Methods, book 6, chap. A49, 6 p., http://dx.doi.org/10.3133/ tm6a49. 


\section{Contents}

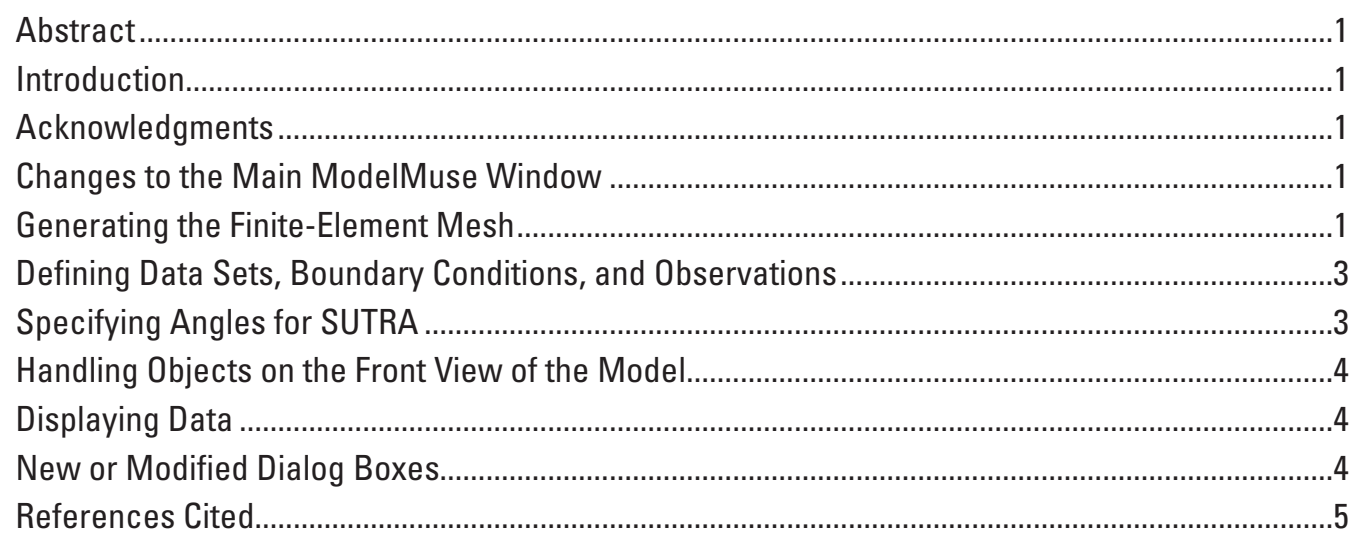

\section{Figures}

1. Example of using objects (colored lines) to define where the mesh should be located. 2

2. Illustration showing the use of fishnet-mesh quadrilaterals to define a mesh.................2

3. Illustration showing the front view of a Saturated-Unsaturated Transport model

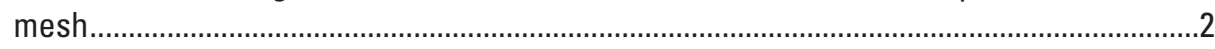

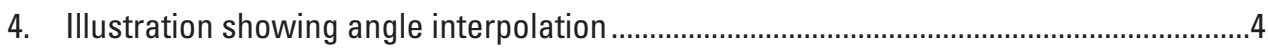

\section{Tables}

1. New or modified dialog boxes in ModelMuse .............................................................. 



\title{
Modifications Made to ModelMuse to Add Support for the Saturated-Unsaturated Transport Model (SUTRA)
}

\author{
By Richard B. Winston
}

\begin{abstract}
This report (1) describes modifications to ModelMuse, as described in U.S. Geological Survey (USGS) Techniques and Methods (TM) 6-A29 (Winston, 2009), to add support for the Saturated-Unsaturated Transport model (SUTRA) (Voss and Provost, 2002; version of September 22, 2010) and (2) supplements USGS TM 6-A29. Modifications include changes to the main ModelMuse window where the model is designed, addition of methods for generating a finite-element mesh suitable for SUTRA, defining how some functions should apply when using a finite-element mesh rather than a finitedifference grid (as originally programmed in ModelMuse), and applying spatial interpolation to angles. In addition, the report describes ways of handling objects on the front view of the model and displaying data. A tabulation contains a summary of the new or modified dialog boxes.
\end{abstract}

\section{Introduction}

This document is a supplement to U.S. Geological Survey (USGS) Techniques and Methods (TM) 6-A29 (Winston, 2009). It is written for a reader generally familiar with ModelMuse applications for the modular groundwater flow model (MODFLOW) (Harbaugh, 2005) or PHAST - a computer program for simulating groundwater flow, solute transport, and multicomponent geochemical reactions (Parkhurst and others, 2004), as described in USGS TM 6-A29. The conventions used in USGS TM 6-A29 are also used in this document.

\section{Acknowledgments}

Alden Provost and Clifford I. Voss (USGS), and Jeffrey McKenzie (Department of Earth and Planetary Sciences, McGill University) are gratefully acknowledged for testing and making suggestions for improving ModelMuse. Thanks are also extended to Alden Provost and Clifford I. Voss for their helpful review comments.

\section{Changes to the Main ModelMuse Window}

With MODFLOW and PHAST models, the main ModelMuse window has four panes: a top view, a front view, a side view, and a three-dimensional (3D) view. The front and side views show cross sections parallel to the sides of the finite-difference grid. The finite-difference grid is rectangular in plan view with vertical sides, so it makes sense to display it from the front and side views. SUTRA meshes can be any shape in plan view, so they do not have front and side views. With SUTRA models, the side view is eliminated and the front view can show cross sections at arbitrary angles and positions. Although the name "front view" is now something of a misnomer when used with SUTRA models, there is no better term that can be used for both models with grids and those with meshes; changing the name would only create confusion when used with models with grids. The selection cube on the front view displays the position of the cross section relative to the width of the mesh perpendicular to the cross section.

The Grid menu is replaced by a Mesh menu when ModelMuse is being used with a SUTRA model. The Mesh menu contains items used to create or edit the mesh and to display relevant information. Details are included in the help system for ModelMuse.

\section{Generating the Finite-Element Mesh}

For two-dimensional (2D) SUTRA models, the mesh consists of quadrilateral elements on the top view of the model. For three-dimensional (3D) SUTRA models, the mesh consists of vertically aligned hexahedral elements such that layers of nodes are also vertically aligned. Layers of elements pinch out if the vertical thickness of a layer is less than or equal to zero. Pinchouts can leave holes in the mesh if neither the underlying nor overlying units pinch out.

In earlier versions of ModelMuse, the user could generate a grid with variably spaced rows and columns by using objects to define the desired grid spacing. This can still be done with grid-based models. However, such objects can now be used to 
define the location of the mesh and the desired spacing between nodes in SUTRA models. Such objects must be created on the top view of the model. The object with the largest area defines the area to be included in the mesh (the outline of the model domain). Any polygon objects or sections of objects that are completely enclosed within the domain outline define holes in the mesh (blue lines, fig. 1). To define polygonal areas within the mesh that will not be holes inside the mesh, two or more polylines that cross each other can be used (red lines, fig. 1). The mesh is constrained to follow along the edges of any objects that define the desired node spacing. The user should be aware that there will always be at least one SUTRA node in between any two vertices of an object used to constrain the mesh. A modification of the method described by Sarrate and Huerta (2000) is used to generate the mesh. The primary modification was that Sarrate and Huerta (2000) did not describe how to subdivide a domain automatically when the domain contained intersecting boundaries. The method in ModelMuse automatically identifies polygons that can be created by intersecting boundaries and uses them to subdivide the domain outline.

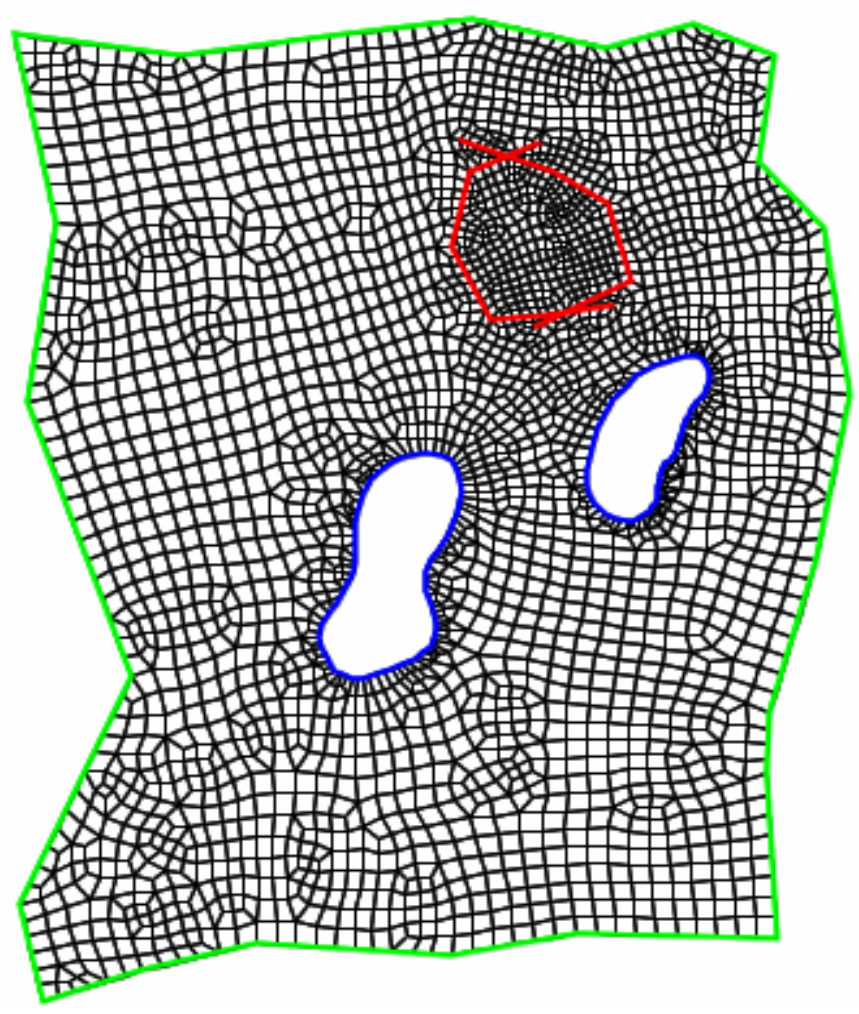

Figure 1. Example of using objects (colored lines) to define where the mesh should be located. The two blue interior objects define holes in the mesh. The two red interior lines that cross each other define an area of mesh refinement that is not a hole in the mesh.

Besides using objects to create the mesh, the user can also draw "fishnet-mesh quadrilaterals" and specify the number of elements to be created along two adjacent edges of the quadrilateral. When the mesh is created in this way, linear interpolation along each side and interpolation between opposite sides of the fishnet-mesh quadrilaterals are used to determine the locations of the nodes and elements (fig. 2). The user can select nodes or elements in the mesh to move or delete them and can also manually draw elements.

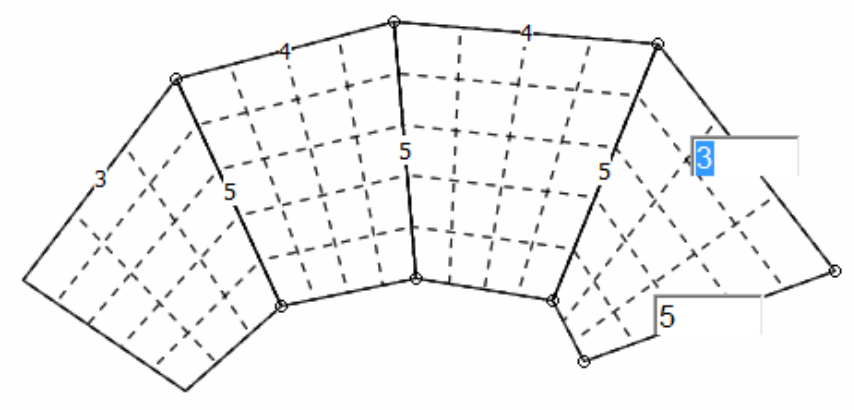

Figure 2. Illustration showing the use of fishnet-mesh quadrilaterals (solid lines) to define a mesh.

The 3D mesh consists of vertically aligned layers of nodes and generalized hexahedral elements. For 3D meshes, a cross section of the mesh is displayed on the front view of the model (fig. 3). The user can move the cross section to any location or angle. The edges of elements intersected by the cross-section line on the top view of the model are used to select a series of nodes. These nodes are used to draw a cross section displayed on the front view. The centers of the elements intersected by the cross-section line can also be displayed on the top and front views. Element outlines, cell outlines, node numbers, and element numbers can also be displayed.

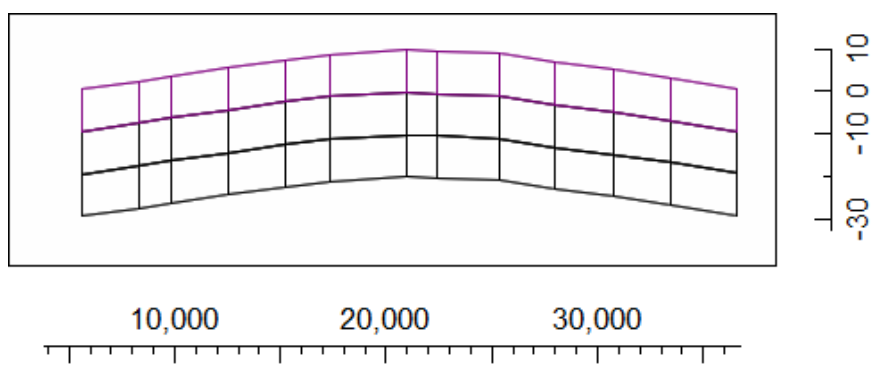

Figure 3. Illustration showing the front view of a SaturatedUnsaturated Transport model (SUTRA) mesh.

The Mesh Information dialog box displays statistics about the mesh. The dialog box also contains tables that show the angles and aspect ratios of $2 \mathrm{D}$ elements and the number of $2 \mathrm{D}$ elements per node. If any elements have invalid shapes, such as concave elements, their numbers are displayed in a 
separate table. The aspect ratio is defined here as the minimum distance from any node to a line connecting two nodes in the same element to which the first node is not adjacent divided by the length of the longer of the two diagonals of the element. When the user clicks on a node or element number in one of these tables, a Go to button appears. Clicking the Go to button moves the top and front view of the model so that the node location or element center is centered in the top and front views of the model.

The SUTRA Layer Groups dialog box is used in 3D models to define the layers in the SUTRA model. Each "Layer Group" defines one or more layers in the model. For each layer group, a data set is defined that is used to specify elevation of nodes on the layer at the bottom of the layer group. Another data set, SUTRA_Mesh_Top, defines the top of the mesh. If a layer group defines multiple layers, a layer spacing specified by the user determines the vertical spacing of the nodes between the top and bottom of the layer group. The bottom of each layer group also defines the top of the layer group beneath it.

\section{Defining Data Sets, Boundary Conditions, and Observations}

In SUTRA models, some data sets are defined at nodes, and others are defined at elements. For a user-defined data set, the user can choose whether the data set is defined at nodes or elements. In ModelMuse, data sets for models with grids have a $3 \mathrm{D}$ array of values. The dimensions correspond to the layers, rows, and columns of the grid. For SUTRA models, the same structure is used but the data sets always have a single row. For 2D models, the node or element number is treated as the column number of the data set and the data set will have one row and one layer. For 3D data sets, the column number is the same as if the mesh were a 2D mesh and the number of rows is still one; however, the number of layers in the data set is the same as the number of layers of nodes or elements.

The data sets required by SUTRA will vary depending on the options selected by the user. ModelMuse automatically creates any data sets required by SUTRA. If a previously created data set is no longer required because of a change in the user-selected options, the data set is not deleted but is marked by ModelMuse as "optional." Any formulas that use such optional data sets continue to be valid.

Objects can be used to set values of data sets in the same way as with MODFLOW and PHAST models. As in PHAST, an object can only be used to set values of data sets that are evaluated at nodes if the object is also evaluated at nodes. Likewise, an object can only be used to set values of data sets that are evaluated at elements if the object is also evaluated at elements.

As configured for SUTRA models, the Object Properties dialog box in ModelMuse contains a tab labeled SUTRA Features. Controls in it are used to define observations and boundary conditions. Because the boundary conditions are defined at nodes, only objects that are evaluated at nodes can be used to define boundary conditions. Observations, however, can be defined with any object. With both observations and boundary conditions, the user can either select a previously defined time schedule or specify the times for a "custom" time schedule. ModelMuse aggregates all the times in all the time schedules - both those that have been explicitly defined and "custom" time schedules, to define any time schedules required by SUTRA. If required, time-cycle and step-cycle schedules will be modified to time lists to ensure that the times specified by the user are included correctly in the time schedules.

In SUTRA, observation locations are selected by the user by specifying the coordinates of the observations. These may or may not be at a node. In ModelMuse, observation locations that are not at nodes in 2D models are selected by creating an object consisting only of point sections. Objects with sections that are not lines or polygons will define observations at nodes or at the centers of elements, depending on whether the object is evaluated at nodes or elements. For 3D models, the treatment is the same, except that objects specifying observations that are not at nodes must only have one (and only one) third dimension formula. For observations, the user must specify the observation time or times.

For boundary conditions in ModelMuse, the user must specify the times at which the boundaries are activated or deactivated, or at which changes to the boundary values are made. If a boundary is active, the user must specify either one or two additional values, depending on the type of boundary.

For specified fluid-flux boundaries, for example, the user must set both the fluid flux and either the concentration or temperature, whereas for a specified concentration boundary, only the specified concentration needs to be set. ModelMuse allows the user to set these values either with numbers or formulas.

\section{Specifying Angles for SUTRA}

In SUTRA, for 3D models, the user must specify three angles to set the direction of the maximum, middle, and minimum permeability, and the longitudinal and transverse dispersivity values associated with those permeability directions (one angle for 2D models). ModelMuse makes specifying such angles easier; the user can draw objects on the top view of the model and use the function "ObjectCurrentSegmentAngleDegrees" to specify the angle. The angle that is assigned is the angle of the segment of the object that is being evaluated. Furthermore, the interpolation methods in ModelMuse have been altered to interpolate angles in a different way from other data types. If the units of the data set have been set to degrees or radians, when interpolation is performed on the data, the values to be interpolated are not the angles themselves but rather the sine and cosine of the angles. The interpolated values of sine and cosine are treated as two 
edges of a right triangle and the angle of the hypotenuse of the triangle is the interpolated angle. In figure 4 , the four objects in blue are all used to assign the maximum permeability angle by interpolation. All four objects use the formula "ObjectCurrentSegmentAngleDegrees"; however, in figure $4 A$, the data set is assigned units of "Degrees," whereas in figure $4 B$, no units are assigned. The goal is to have all the vectors point outward from the center of the mesh. This goal is achieved in figure $4 A$ but not in figure $4 B$.

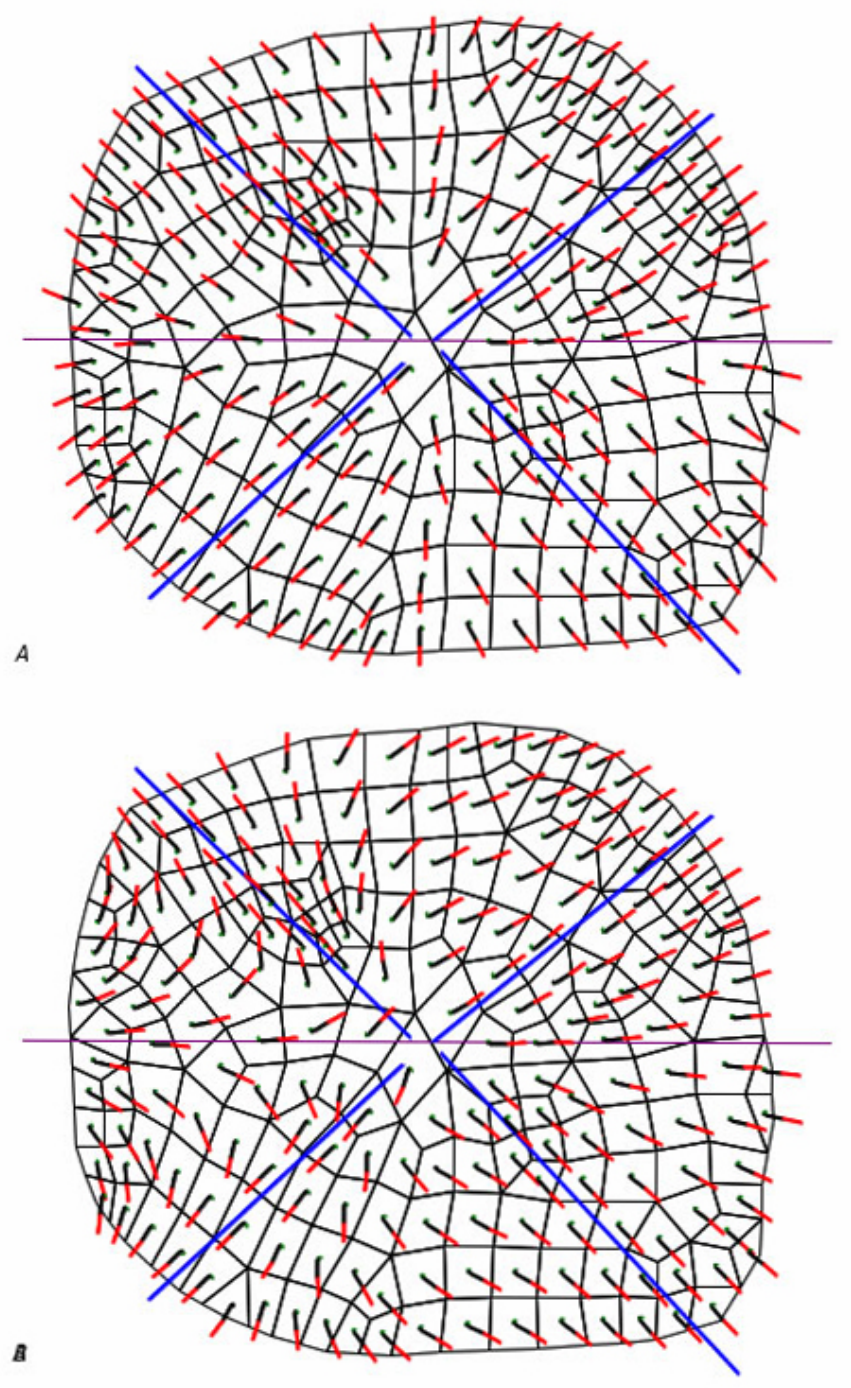

Figure 4. Illustration showing angle interpolation. In both illustrations, the blue objects specify ANGLE1 by interpolation using the formula "ObjectCurrentSegmentAngleDegrees" and the vectors display the maximum permeability. $A$, The data set has its unit set to "Degrees" so that a special form of interpolation is used. $B$, The data set has no units so the ordinary form of interpolation is used.

\section{Handling Objects on the Front View of the Model}

In SUTRA models, objects drawn on the front view of the model are only visible when the angle of the cross section is the same as the angle that the cross section was set to when the object was created. In the Show or Hide Objects dialog box, an icon is displayed before the names of objects drawn on the front view of the model. The icon indicates the angle of the cross section needed to display the object and whether or not the cross section is at that angle. Clicking on the icon will rotate the cross section to the angle required to display the object, if it is not already at that angle.

\section{Displaying Data}

ModelMuse with SUTRA can display data by coloring the mesh or drawing contour lines, similar to the way this is done by ModelMuse with MODFLOW and PHAST. ModelMuse can also display vectors for SUTRA (fig. 3). Two types of vectors can be displayed:

- When velocities are imported from model results, the velocities can be displayed as vectors.

- The maximum, middle, and minimum permeabilities and dispersivities can be displayed as vectors with the maximum, middle, and minimum values each displayed with separate colors.

All vectors are displayed in two colors. The base of the vector is at the center of the element. The first half of the vector from its base to middle is displayed in black. The second half is displayed in a color specified by the user.

The 3D view of the model, by default, displays the element outlines on the selected layer and the cross section. It can also display vectors and cells or elements colored according to the values of a data set.

\section{New or Modified Dialog Boxes}

There are 14 new dialog boxes that are specific to SUTRA, and 5 others have been modified to include information that is also specific to SUTRA. These are listed in table 1. The Help system for ModelMuse contains more information about these dialog boxes. 


\section{References Cited}

Harbaugh, A.W., 2005, MODFLOW-2005, the U.S. Geological Survey modular ground-water model - the ground-water flow process: U.S. Geological Survey Techniques and Methods, book 6, chap. A16, 253 p., http://pubs.usgs.gov/tm/2005/tm6A16/.

Parkhurst, D.L., Kipp, K.L., Engesgaard, Peter, and Charlton, S.R., 2004, PHAST - A program for simulating ground-water flow, solute transport, and multicomponent geochemical reactions: U.S. Geological Survey Techniques and Methods, book 6, chap. A8, 154 p., http://pubs.usgs.gov/tm/2005/tm6A8/.

Sarrate, Josep, and Huerta, Antonio, 2000, Efficient unstructured quadrilateral mesh generation: International Journal of Numerical Methods in Engineering, v. 49, p. 1327-1350.
Voss, C.I., and Provost, A.M., 2002 (version of September 22, 2010), SUTRA, A model for saturated-unsaturated, variabledensity ground-water flow with solute or energy transport: U.S. Geological Survey Water-Resources Investigations Report 02-4231, 291 p., http://water.usgs.gov/nrp/ gwsoftware/sutra/SUTRA_2_2-documentation.pdf.

Winston, R.B., 2009, ModelMuse-A graphical user interface for MODFLOW-2005 and PHAST: U.S. Geological Survey Techniques and Methods, book 6, chap. A29, 52 p., http://pubs.usgs.gov/tm/tm6A29/. 
Table 1. New or modified dialog boxes in ModelMuse.

\begin{tabular}{|c|c|c|}
\hline Dialog box name & Command used to display dialog box & Purpose of new dialog box or of modification \\
\hline Import SUTRA Model Results & File|Import|Model Results... & $\begin{array}{l}\text { New. Import pressure, concentration or } \\
\text { temperature, saturation, and groundwater } \\
\text { velocities calculated by SUTRA. }\end{array}$ \\
\hline Mesh Generation Control Variables & Mesh|Generate Mesh... & $\begin{array}{l}\text { New. Specify the mesh generation method } \\
\text { and the values of parameters used to } \\
\text { control finite-element mesh generation. }\end{array}$ \\
\hline Mesh Renumbering Method & Mesh|Renumber Mesh & $\begin{array}{l}\text { New. Specify the method used to renumber } \\
\text { the nodes and elements in the mesh. }\end{array}$ \\
\hline Specify Mesh & $\begin{array}{l}\text { Mesh|Specify Mesh... or Mesh|Specify } \\
\text { Fishnet-Mesh Quadrilaterals }\end{array}$ & $\begin{array}{l}\text { New. Numerically specify all the nodes and } \\
\text { elements in either the mesh or the fishnet- } \\
\text { mesh quadrilaterals. }\end{array}$ \\
\hline Fishnet Quadrilateral Properties & Double-click on a fishnet-mesh quadrilateral. & $\begin{array}{l}\text { New. Specify the locations of the nodes in a } \\
\text { fishnet-mesh quadrilateral and the spacing } \\
\text { of nodes along the edge of a fishnet-mesh } \\
\text { quadrilateral. }\end{array}$ \\
\hline Node Location & $\begin{array}{l}\text { Press the Move or delete nodes or elements } \\
\text { button and then double-click on a node } \\
\text { or element. }\end{array}$ & $\begin{array}{l}\text { New. Numerically specify the locations of } \\
\text { selected nodes. }\end{array}$ \\
\hline Data Visualization & Data|Data Visualization & $\begin{array}{l}\text { Specify vectors of velocity, permeability, or } \\
\text { dispersivity. }\end{array}$ \\
\hline Data Sets & Data|Edit Data Sets... & $\begin{array}{l}\text { Specify degrees or radians, as the units affect } \\
\text { how interpolation is performed. }\end{array}$ \\
\hline Customize SUTRA Mesh & View|Customize SUTRA Mesh... & $\begin{array}{l}\text { New. Change how the mesh is displayed, } \\
\text { including element outlines, node numbers, } \\
\text { and element numbers. }\end{array}$ \\
\hline SUTRA Options & Model|SUTRA Options & $\begin{array}{l}\text { New. Specify a variety of nonspatial data for } \\
\text { SUTRA. }\end{array}$ \\
\hline SUTRA Layer Groups & Model|SUTRA Layer Groups & $\begin{array}{l}\text { New. Specify the layer structure for 3D } \\
\text { SUTRA models. }\end{array}$ \\
\hline SUTRA Time Controls & Model|SUTRA Time Controls & New. Specify time schedules for SUTRA. \\
\hline SUTRA Output Control & Model|SUTRA Output Control & New. Specify the type of SUTRA output. \\
\hline SUTRA Program Location & Model|SUTRA Program Location & $\begin{array}{l}\text { New. Specify the location of the SUTRA } \\
\text { program. }\end{array}$ \\
\hline
\end{tabular}




\section{$\frac{\mathbb{3}}{3}$}

ISSN 2328-7055 (online)

http://dx.doi.org/10.3133/tm6a49

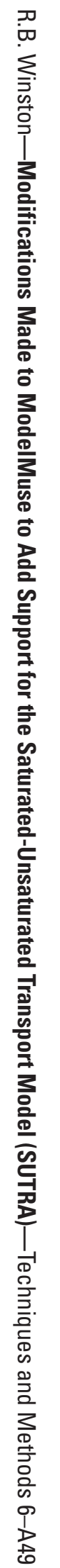

